\title{
Prescriptive Maintenance: Building Alternative Plans for Smart Operations
}

\author{
Henrique Marques and Alessandro Giacotto \\ AerologLab-ITA, Aeronautics Institute of Technology, São José dos Campos, São Paulo/Brazil \\ E-mail: hmarques@ita.br, agiacott@ita.br
}

\begin{abstract}
Smart operations require the ability to generate alternative plans whenever a change in operations occurs in an unplanned manner. Alternate maintenance plans, in a highly dynamic context such as airline operations, require the ability to foresee small developments in terms of labor allocation, repairable items, and downtime, when and where they were not previously scheduled. In addition to being able to cause the disruption of the air transport network and consequent financial losses, it causes loss of trust in the company brand. Prescriptive maintenance is a potential technological response when using Artificial Intelligence to suggest alternative plans promptly so that decisionmakers can reduce the impact on air operations. This paper proposes a framework for the construction of an integrated prescriptive maintenance solution that is certifiable by using auditable methods and extensible to complex systems of other industries. The adoption of prescriptive maintenance not only enhances the use of health management systems, widely available in modern aircraft fleets that have the potential to predict the remaining useful life of items of interest, but also allows identifying more than one response alternative to conflicts of interest in the conduction of the smart operations of air transport companies.
\end{abstract}

Keywords: Prescriptive Maintenance, Artificial Intelligence, e-Maintenance, Smart Operations, Information Fusion, Diagnosis, Prognosis

\section{Introduction}

The aircraft maintenance schedule is among the major decisions an airline has to make during its operation since it directly affects maintenance costs and aircraft availability. The development of aircraft maintenance schedule is a complicated task involving the combination of a variety of parameters such as mechanic and engineering maintenance labor, consumable materials and equipment spare logistics, and, from the operator point of view, the demand for air transportation [1]. On the other hand, more than $80 \%$ of passengers are now traveling on tickets priced at less than base fare and this downward pressure on revenues is leading many carriers to focus their attention on controlling maintenance costs while ensuring safety and airworthiness, complying thus with regulators requirements.

The predictive maintenance philosophy uses sensors and other monitoring strategies to gauge when complex systems actually will require maintenance. It is condition-based and relies on real-time health monitoring data available embedded in the system of interest. Data analytics procedures calculate the remaining useful life (RUL) of the equipment, allowing the operator to perform the maintenance before the faults happen, as the same philosophy of preventive maintenance, but not in a predetermined period (or cycles). The idea is to be able to establish the opportunistic moment to make the maintenance, postponing, or anticipating the moment, causing less downtime.

The prescriptive maintenance philosophy is a step ahead providing real-time adaptive recommendations, using artificial intelligence, about the tasks that should be done and keep updating as the operation continues. In other words, the prescriptive maintenance not only is based on the failures' prediction accordingly to the analysis of data patterns and trends, but also taking the specific company's maintenance process into consideration to provide detailed recommendations, and supports the solution-finding process.

This paper proposes a framework to tackle the scheduling maintenance problem adopting prescriptive maintenance strategy, which has to be certifiable, auditable, customized at the aircraft level [2], comprised of key indicators to measure its efficiency, capable of an adaptive context-awareness system and extensible to other industries.

\section{Literature review}

According to [3], key performance indicators are initially considered during the systematic inspection of failure patterns to understand their technical characteristics, criticality (downtime, costs, and occurrence) and their effects 
on maintenance indicators. Besides the distinct task of timely executing a maintenance order, the measurement and control of the success of predictions and useful recommendations are not only used for the refinement of a machine learning method and a decision model, but also for the calculation of maintenance key performance indicators. By quantifying the defined key indicators, the current state maturity level is assessed, and weaknesses are identified. These results serve as a basis for the definition of company-specific target maturity state based on its strategic priorities.

As stated in [4], a feedback mechanism has to be added so that the recommendations are continuously improved based on cost-related sensor-generated data during action implementation. In this way, the phases of diagnostics, prognostics, and recommendations can be further investigated and enriched with new and improved algorithms.

Prescriptive maintenance is context-aware by nature. According to [5], in the prescriptive maintenance solutions proposed so far, the maintenance operators have to accept or reject the suggested tasks since their implicit experiential and "tribal" knowledge is necessary to a context-dependent maintenance decision, in a highly flexible operational environment. The information regarding the acceptance or rejection of the suggested tasks is then processed and analyzed as feedback information to improve the defined rules and measures continuously. In this sense, work is required to automatically adapt the proposed prescriptive maintenance set of rules to a dynamic operational environment, allowing the entire maintenance process to be less reliant on human knowledge and experience through machine learning approaches.

The prescriptive maintenance looks to the maintenance process powered by the Internet of Things (IoT), and its value is on the non-routine maintenance tasks when talking about heavy maintenance and in the challenge coming from the fleet schedule evolution in terms of line maintenance tasks. In terms of maintenance planning, while the Condition Based Maintenance (CBM) approach looks to the "when" and "why" to maintain, the prescriptive maintenance looks to the "what" and "where", defining the place and order of the maintenance tasks to accomplish and "who" should be engaged to execute each one [6]. To prescribe the way the tasks should be done is necessary to have inputs such as the MRO processes, available GSEs, spares, manpower and the condition of the system which is receiving the maintenance resources availability status should be provided in real-time through the IoT. In this sense, the prescriptive maintenance is not a substitute or option to the Reliability-Centered Maintenance (RCM) paradigm, but the next step to its complete implementation on the field.

As mentioned by [7], nowadays, preventive maintenance does not consider operational contexts, such as environmental parameters, and these strongly affect components' lifetime. The ever-growing dynamism of the operational environment has also spurred the exploration of flexible on-line optimization approaches capable of reworking maintenance schedules incrementally (also known as rescheduling) to accommodate the contextual variability of the operation in different aspects.

When turning the focus to prescriptive maintenance, the most challenging paradigm encountered in practice remains tightly coupled to the match between the formulated optimization problem and the decision-making process that such a problem aims to model. Industries are complex environments where humans and machinery coexist and interact, often without holistically centralized management. It is often the case that actions triggered by a predictive model do not conform to the practical criteria or constraints under which such actions would be manually enforced, like time and cost. The developed models would fail to apply when deployed.

New working methodologies are needed to ensure that the hypothesis is aligned with the real operational requirements. Besides, such methods should also account for other practical aspects including the variability of metrics and constraints along time, cost implications of decisions made by the model, or the presence of conflicting objectives in the criteria guiding such choices.

In addition to it, as mentioned by [8], although the prescriptive maintenance concepts deliver promising results, the generalizability of the approach has not been tested yet and, as future work, the concept shall be validated in different industries to test its generalizability.

\section{Prescriptive maintenance framework}

In this section, the authors propose the framework, from now on called Smart Prescriptive Maintenance Framework (SPMF) that can be used to build an effective prescriptive maintenance program for a fleet of commercial aircraft. The section starts with a description of the framework structure, then goes on with the description of the key enablers' elements and technologies requirements, continues with the description of key indicators to measure the efficiency of the framework, and describes the requirements of the adaptiveness and generalizability.

\subsection{The SPMF framework}

The framework is built on three domains of interest: The system's reliability, availability, maintainability, and safety (RAMS) factors, the operating environment in which the system is operated, and the maintenance environment that will perform the maintenance tasks required to restore the system to the necessary operating conditions, within its intended reliability characteristics (see fig. 1). Time and cost are treated as constraints in all domains.

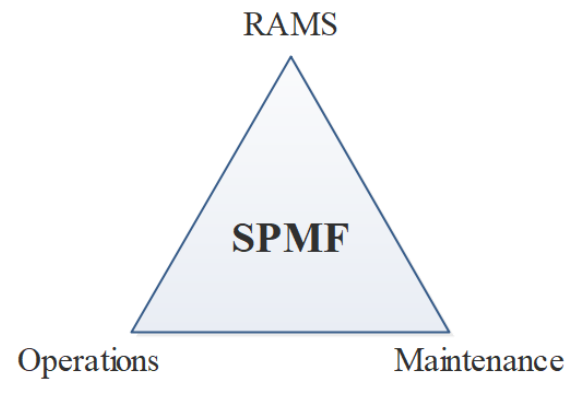

Figure 1: Framework domains of interest. 
Each domain has essential information that will feed the framework through the various phases of information fusion until a group of maintenance actions is defined. The SPMF fusion flow diagram describes how the framework works as well as the inputs needed and the outputs expected in each phase.

As shown in fig. 2, the flow of information fusion is based on the capability to make the diagnosis, identifying the next operational demands to achieve the prognosis and based on

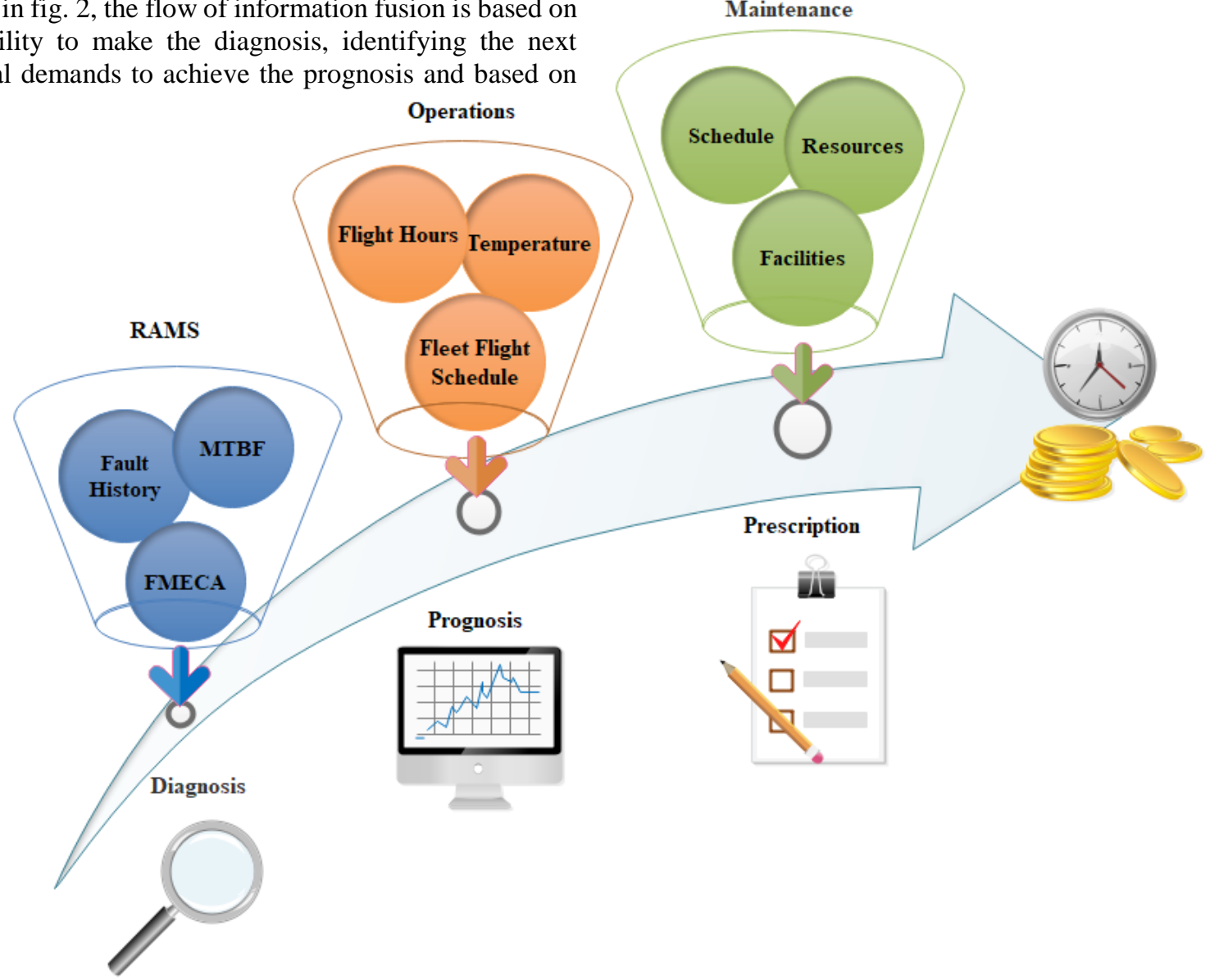

Figure 2: Framework fusion methodology.

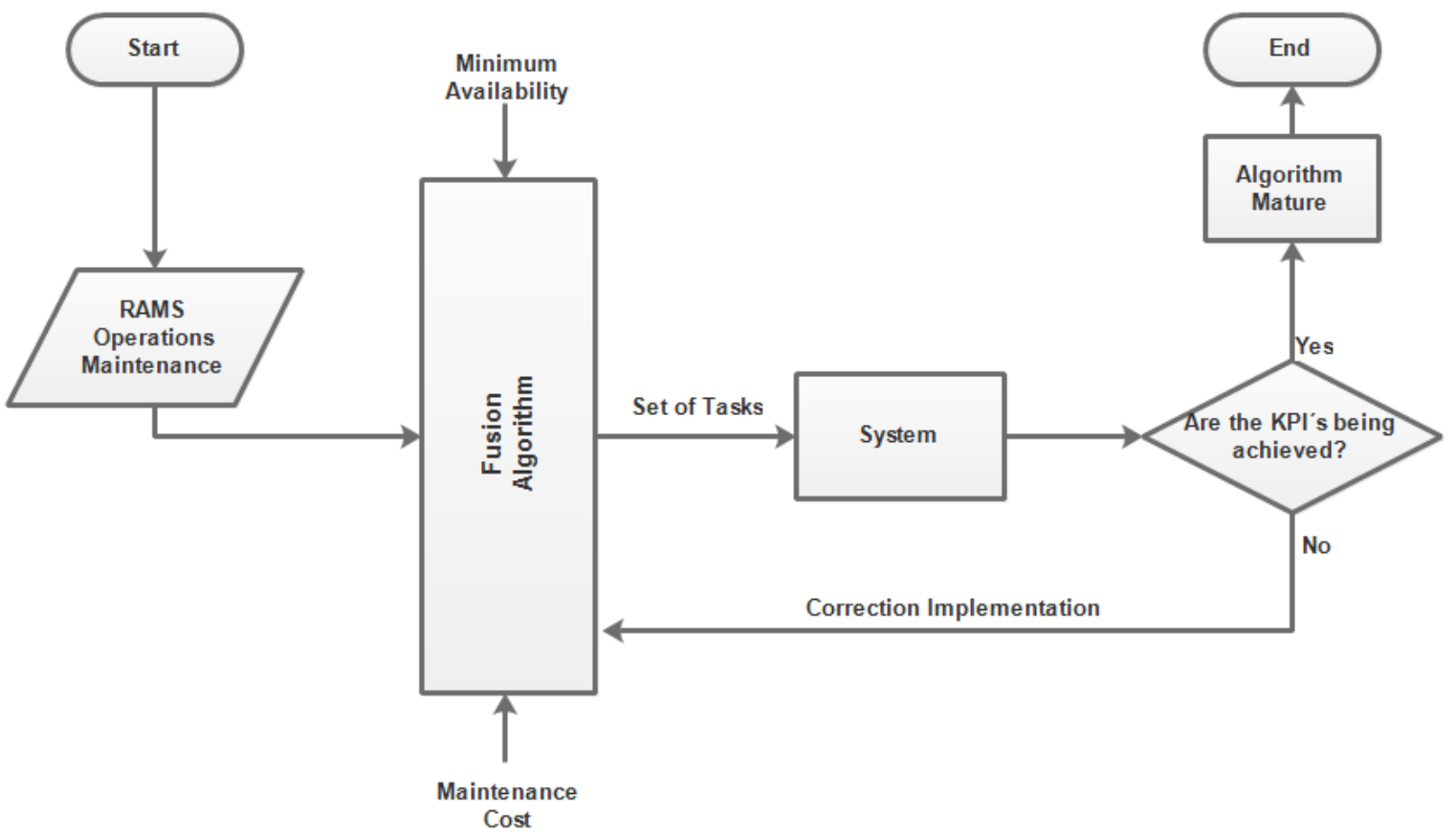

Figure 3: Framework workflow. the maintenance capabilities, the prescription of the set of maintenance tasks.

From another perspective, the framework is constituted by five building blocks: inputs, fusion algorithm, output, the supported system, and the efficiency check process, as shown in fig. 3.

Maintenance 


\subsection{Inputs}

The inputs building block comprises the information needed to feed the fusion algorithm. Table 1 describes a simplified list of information from the RAMS, Operations, and Maintenance domains.

\subsection{Fusion algorithm and its output}

The fusion algorithm proposed is an Artificial Intelligence (AI) problem-solving agent [9]. Once all the inputs are informed, the algorithm starts to search for the most probable effective action or sequence of actions to solve the maintenance problem. Once the actions are selected, the algorithm recommends the best scheduling according to the minimum fleet availability requirement and the constraint of the direct (DMC) and indirect maintenance cost (IMC), providing, thus, the output of the fusion step.

\subsection{Efficiency check}

After the implementation of the tasks over the system, the algorithm itself verifies, during the efficiency check, if the maintenance actions are practical, the fleet availability is according to customer's requirement and maintenance costs minimized. If all these requirements are satisfied, then the selection of the same actions is reinforced, and the algorithm is considered mature. If at least one of the above conditions is not satisfied, then the probability attached to each task is redistributed, and a new set of actions is proposed in the next iteration.

\subsection{Key indicators}

As previously mentioned, the performance of the system is checked against two requirements: fleet availability and minimization of the system's maintenance cost. Both parameters are also key indicators of the algorithm performance: is the fleet availability above the threshold aligned with the operator's intent? Are the DMC and IMC contained in comparison to historical maintenance cost or operator estimates?

\subsection{Adaptiveness and context awareness}

Adaptiveness and context awareness are essential characteristics of the SPMF. These characteristics allow prescriptive maintenance generation specifically for each "tail number". Some inputs, for example, the number of flight hours (FH) or flight cycles (FC), temperature \| humidity, tribal knowledge, RUL and fault history, are a function of each operational environment, that is, one aircraft presents different values from another, and the same happens to maintenance teams and MROs. The prescriptive approach favors the utilization of the capability to identify the individual characteristics of each system, instead of using their average performance.

Thus, this approach makes use of some enabling technologies present in the industry 4.0 such as networking, availability of extensive data collected by sensors and the use of efficient algorithms necessary to support real-time maintenance execution monitoring [10].

Table 1: Framework inputs

\begin{tabular}{|c|c|}
\hline Input & Description \\
\hline Manufacturer Maintenance Plan & $\begin{array}{l}\text { Initial maintenance plan provided by the manufacturer at the aircraft entry into } \\
\text { service. It is developed according to MSG-3 principles, and it is updated yearly } \\
\text { depending on the commercial agreement between the airliner and the operator }\end{array}$ \\
\hline Flight Hour \| Flight Cycle & $\begin{array}{l}\text { The number of flight hours (FH) or flight cycles }(\mathrm{FC}) \text { represent the usage of the } \\
\text { aircraft, system or specific equipment. These parameters are useful since, very } \\
\text { often, the reliability of the equipment is a function of the system usage }\end{array}$ \\
\hline Temperature || Humidity & $\begin{array}{l}\text { Environmental parameters have to be considered since operational conditions } \\
\text { influence the failure rate of the equipment in use }\end{array}$ \\
\hline Remaining Useful Life & $\begin{array}{l}\text { The remaining useful life (RUL) curve of each aircraft of the fleet should be } \\
\text { predicted, based on the expected fleet flight schedule. Thus, some prognostics and } \\
\text { health management (PHM) system has to be present in the aircraft to help plan the } \\
\text { maintenance schedule. If not present, the fusion algorithm would rely on fault } \\
\text { history, making forecastings }\end{array}$ \\
\hline "Tribal" Knowledge & $\begin{array}{l}\text { "Tribal" knowledge is any information gathered from experience and lessons } \\
\text { learned by the team who is responsible for the maintenance execution. This subset } \\
\text { of data is often unstructured }\end{array}$ \\
\hline Fault History & Historical data of all the equipment faults \\
\hline Failure Mode and Criticality Analysis & $\begin{array}{l}\text { Failure mode and criticality analysis (FMECA) particularly helpful to support } \\
\text { inductive AI approaches }\end{array}$ \\
\hline Mean Time Between Failures & $\begin{array}{l}\text { The mean time between failures (MTBF) represents the expected time between } \\
\text { failures of a system, during normal operation }\end{array}$ \\
\hline Minimum Equipment List & $\begin{array}{l}\text { It details which equipment is allowed to be inoperative without grounding } \\
\text { the aircraft }\end{array}$ \\
\hline Maintenance Cost & $\begin{array}{l}\text { The maintenance cost, which includes man-hour and material related to all aircraft } \\
\text { maintenance activities }\end{array}$ \\
\hline Fleet Flight & ne schedule of all the company's flights to be accomplished \\
\hline
\end{tabular}




\subsection{Extensibility to other industries}

Ideally, the SPMF algorithm can be used in those fields where a complex system is in play. The critical point, in this case, is the adoption of parameters that can describe the specific complex system accurately. The current development proposes to extend the SPMF to the medical field (MedicalSPMF), being the human body the complex system to be "supported". Table 2 suggests, for each input listed in the tab. 1 , the respective information applicable for the MedicalSPMF.

Table 2 - Medical SPMF inputs

\begin{tabular}{ll}
\hline Aircraft complex system input & Human body complex system input \\
\hline Manufacturer Maintenance Plan & Formally recommended checks and their frequency \\
\hline Flight Hour || Flight Cycle & Age \\
\hline Temperature || Humidity & Body temperature \\
\hline Remaining Useful Life & Prognostics \\
\hline "Tribal" Knowledge & Informal lessons learned of the medical team \\
\hline Fault History & Patient health history \\
\hline Failure Mode and Criticality Analysis & Medical knowledge related to the specific organs failures being treated \\
\hline Mean Time Between Failures & Not applicable \\
\hline Minimum Equipment List & Medical statistics knowledge related to the organs failures being treated \\
\hline Direct Maintenance Cost & Cost of treatment \\
\hline
\end{tabular}

The prescriptive maintenance has been deployed in industrial plants [11] and elevators and escalators maintenance [12] with improved results, but still in its early stages of development.

\section{Conclusion and future work}

This work proposes the Smart Prescriptive Maintenance Framework (SPMF) as a structure to implement an AI, context-aware, adaptive algorithm to support a fleet of commercial jets. The framework includes efficiency checks against operator requirements such as the fleet availability and the direct maintenance cost, and depends on specific operational, engineering and economic data to be assertive such as: manufacturer maintenance plan, $\mathrm{FH}, \mathrm{FC}$, temperature, humidity, RUL, "tribal" knowledge, fault history, FMECA, MTBF, MEL and DMC, among others.

The SPMF concepts apply to all industries where a complex system has to be supported, and in this paper is mentioned the extension to the medical field, as the human body the system to be supported. In this sense, it is recommended the adoption of suitable inputs and requirements for the treatment of a human body.

Some essential questions and open technical challenges have been identified, related to the AI modeling and the right metaheuristics to be used. It also remains to be clarified if there are key performance indicators other than cost and fleet availability to drive the prescriptive maintenance and check its efficiency. Regarding the inputs data, it is also unquestionable that such information will have to be consolidated in an interoperable data standard to be determined. Referring to the medical field still has to be addressed the best inputs to be adopted.

The next steps refer to the determination of the best metaheuristics to be used by the SPMF, identification of the most suitable KPI's and test of the algorithm framework in a real fleet. Successively, once the algorithm is mature and the right inputs are identified, tests will be conducted in the medical field to verify the extensibility of the SPMF concepts.

\section{References}

[1] C. Sriram and A. Haghani, "An optimization model for aircraft maintenance scheduling and re-assignment," Transportation Research Part A: Policy and Practice, vol. 37, no. 1, pp. 29-48, 2003.

[2] Federal Aviation Administration, "Advisory Circular 43-218 Integrated Aircraft Health Management (Draft)," 09-Jul-2019. [Online]. Available: https://webcache.googleusercontent.com/search?q=cach e:jOGamJpbqB4J:https://www.faa.gov/aircraft/draft_do cs/media/afx/AC_43-

218_Coord_Copy.pdf $+\& c d=1 \&$ hl $=$ pt-

BR\&ct=clnk\&gl=br. [Accessed: 19-Sep-2019].

[3] T. Nemeth, F. Ansari, W. Sihn, B. Haslhofer, and A. Schindler, "PriMa-X: A reference model for realizing prescriptive maintenance and assessing its maturity enhanced by machine learning," Procedia CIRP, vol. 72, pp. 1039-1044, 2018.

[4] A. Bousdekis, N. Papageorgiou, B. Magoutas, D. Apostolou, and G. Mentzas, "Enabling condition-based 
maintenance decisions with proactive event-driven computing," Computers in Industry, vol. 100, pp. 173183, 2018.

[5] K. Matyas, T. Nemeth, K. Kovacs, and R. Glawar, "A procedural approach for realizing prescriptive maintenance planning in manufacturing industries," CIRP Annals, vol. 66, no. 1, pp. 461-464, 2017.

[6] S. K. editor contributing, "RxM: What is prescriptive maintenance, and how soon will you need it?," Plant Services. [Online]. Available: https://www.plantservices.com/articles/2017/rxm-whatis-prescriptive-maintenance-and-how-soon-will-youneed-it/. [Accessed: 23-Sep-2019].

[7] A. Diez-Olivan, J. Del Ser, D. Galar, and B. Sierra, "Data fusion and machine learning for industrial prognosis: Trends and perspectives towards industry 4.0," Information Fusion, vol. 50, pp. 92-111, 2019.

[8] M. Abramovici, P. Gebus, J. C. Göbel, and P. Savarino, "Provider-Driven Knowledge Allocation Concept for Improving Technical Repair Tasks in IPS2 Networks," Procedia CIRP, vol. 64, pp. 381-386, 2017.

[9] S. Russell and P. Norvig, "AI a modern approach," Learning, vol. 2, no. 3, p. 4, 2005.

[10] A. Ceruti, P. Marzocca, A. Liverani, and C. Bil, "Maintenance in Aeronautics in an Industry 4.0 Context: The Role of Augmented Reality and Additive Manufacturing," Journal of Computational Design and Engineering, 2019.

[11] P. Deka, "Predictive and Prescriptive maintenance of manufacturing industry with machine learning," Medium, 25-Dec-2018. [Online]. Available: https://towardsdatascience.com/predictive-andprescriptive-maintenance-for-manufacturing-industrywith-machine-learning-2078afa76bfb. [Accessed: 23Sep-2019].

[12] ThyssenKrupp Elevator, "MAX - Predictive maintenance solution - ThyssenKrupp Elevator." [Online]. Available: https://max.thyssenkruppelevator.com/en/. [Accessed: 23-Sep-2019]. 